\title{
Long non-coding RNA MALAT1 plays a protective role in bronchopulmonary dysplasia via the inhibition of apoptosis and interaction with the Keap1/Nrf2 signal pathway
}

\author{
Min Zhang, Xiaoyue Zhang, Xiaoyun Chu, Lihua Cheng, Cheng Cai \\ Department of Neonatology, Shanghai Children's Hospital, Shanghai Jiao Tong University, Shanghai, China \\ Contributions: (I) Conception and design: All authors; (II) Administrative support: All authors; (III) Provision of study materials or patients: All \\ authors; (IV) Collection and assembly of data: All authors; (V) Data analysis and interpretation: All authors; (VI) Manuscript writing: All authors; (VII) \\ Final approval of manuscript: All authors. \\ Correspondence to: Cheng Cai. Department of Neonatology, Shanghai Children's Hospital, Shanghai Jiao Tong University, Shanghai 200062, China. \\ Email: caicheng2004@163.com.
}

\begin{abstract}
Background: Bronchopulmonary dysplasia (BPD) is a common respiratory disease in premature infants and is characterized by alveolar and pulmonary vascular dysplasia. Long-term oxygen exposure can cause BPD in preterm infants. Numerous studies have shown that long non-coding ribonucleic acid (lncRNA) is involved in the process of biological metabolism; however, its role in the development of BPD is unclear. Apoptosis-induced factor (AIF) is a key component involved in apoptosis. The Kelch-like ECH-associated protein 1/nuclear factor erythroid-2-related factor 2 (Keap1/Nrf2) signaling pathway is a body-derived antioxidant signaling pathway.

Methods: In this study, the relative expression of metastasis-associated lung adenocarcinoma transcript 1 (MALAT1), AIF, Keap1, and Nrf2 was detected by real-time polymerase chain reaction (PCR). Also, the apoptosis of A549 cells was detected by flow cytometry.

Results: The results showed that, compared to the control group, the expression of MALAT1 increased significantly, and AIF decreased substantially in BPD premature infants. In the A549 hyperoxic lung injury model, compared with the air group, the expression of MALAT1 in the hyperoxia group decreased markedly, while the expression of Keap1 and Nrf2 increased considerably. Furthermore, compared with the control plasmid transfection air group (NC group), the expression of Keap1 and Nrf2 increased significantly in the small interfering RNA (siRNA) group.

Conclusions: These results indicate that MALAT1 can play a protective role in BPD via the reduction of apoptosis and anti-oxidation, offering clinicians a new way to prevent and treat BPD.
\end{abstract}

Keywords: Bronchopulmonary dysplasia; metastasis-associated lung adenocarcinoma transcript 1 (MALAT1); apoptosis induced factor; Keap1; Nrf2

Submitted Jul 18, 2020. Accepted for publication Dec 18, 2020.

doi: $10.21037 /$ tp-20-200

View this article at: http://dx.doi.org/10.21037/tp-20-200

\section{Introduction}

Bronchopulmonary dysplasia (BPD) is one of the most common chronic respiratory diseases in premature infants, and its pathogenesis is based on genetic susceptibility. High-risk factors, such as oxygen therapy, barotrauma, and inflammatory reaction, can damage premature infants' immature lungs, leading to the abnormal repair of lung tissue and abnormalities in lung structure and function (1). Recently, with the application of prenatal glucocorticoids and postnatal pulmonary surfactants, various auxiliary respiratory devices have been updated, and an increasing number of very early premature infants (with gestational 
ages of 22 to 28 weeks and/or birth weights of 501 to $1,500 \mathrm{~g})$ survive. However, the prevalence of premature babies has also increased (2). BPD imposes heavy burdens on families and healthcare systems, and there remains a lack of effective prevention and treatment measures. Thus, preventing and reducing the occurrence of BPD is critically important.

Non-coding ribonucleic acids (RNAs) can be divided into small (18-200 nt) and long (>200 nt) non-coding RNA (lncRNA) (3). Metastasis-associated lung adenocarcinoma transcript 1 (MALAT1) is an important member of the lncRNA family; it is approximately 8,700 nt in length and is localized to human chromosome 11q13.1. It belongs to intergenic lncRNA and is one of the most widely studied lncRNAs (4). MALAT1 was first discovered in studies of non-small cell lung carcinoma (NSCLC) (5), and was determined to be closely related to cell proliferation and angiogenesis $(6,7)$. It can inhibit apoptosis by participating in multiple signaling pathways in cells, thereby accelerating the activation and proliferation of tumor cells $(8,9)$, and thus, was considered a prognostic marker for lung cancer metastasis (10). However, the mechanism through which MALAT1 regulates the pathogenesis of BPD remains unclear.

In 1999, Susin et al. (11) discovered and named apoptosisinduced factor (AIF), which is a flavin protein present in the mitochondrial membrane space. AIF performs the functions of redox and electron transfer and can promote apoptosis and maintain the normal structure of cells, which is important for maintaining the normal structure and physiological activities of cells. Recent studies have found that AIF is highly expressed in tumor cells $(12,13)$, and that knockdown of AIF can inhibit the oxidative phosphorylation of cells by decreasing the expression of mitochondrial respiratory chain-associated protein molecules, thereby inhibiting cell growth. Knocking down AIF can also lead to a significant decrease in cellular reactive oxygen species (ROS) levels, ultimately inhibiting cell metabolism (13). AIF is a mitochondrial oxidoreductase and a cell death effector. Its mitochondrial nuclear translocation plays an important role in apoptosis and contributes to cell death (14).

The Kelch-like ECH-associated protein 1/nuclear factor erythroid-2-related factor 2 (Keap1/Nrf2) signaling pathway is critically important in the cellular antioxidant response. Under physiological conditions, Keap1 acts as a binder that binds to Nrf2, which is subsequently degraded by proteinase enzymes. When oxidative stress occurs, Keap1 acts as a sensor of redox reactions, causing its conformation to change, which leads to dissociation from Nrf2 and the eventual transfer of $\mathrm{Nrf} 2$ into the nucleus to bind to antioxidant response element (ARE), thereby exerting antioxidative damage $(15,16)$. Subsequently, Keap1 enters the nucleus and the cytosol along with $\mathrm{Nrf2}$, and proteinase enzymes then degrade Nrf2, leading to a decrease in Nrf2 levels and the termination of activation (17). Since the Keap1/Nrf2 signaling pathway can resist oxidative stress induced by internal and external oxidation and chemical stimulation, it plays a key role in the defense against all kinds of external injuries, and thus is considered to be the most important endogenous antioxidant signaling pathway in the body (18).

In this study, we examined the expression of MALAT1 and AIF in the serum of premature infants with BPD, and explored the relationship between the expression of MALAT1 and AIF in these patients. The relative expression levels of MALAT1, Keap1, and Nrf2 in the two groups were determined by comparing air and hyperoxic environments, before and after inhibition of MALAT1. We also explored the role of MALAT1 and the Keap1/Nrf2 antioxidant pathway in BPD by detecting the apoptosis rate of A549 cells after silencing MALAT1 exposed to hyperoxia.

We present the following article following the Materials Design Analysis Reporting (MDAR) checklist (available at http://dx.doi.org/10.21037/tp-20-200).

\section{Methods}

\section{Ethical Statement}

This study was conducted following the Declaration of Helsinki (as revised in 2013) and was approved by the committee board of the Shanghai Children's Hospital (NO.: 2015RY009-F01). Informed consent was obtained from the parents of all included patients.

\section{Subjects}

A prospective case-control study was conducted to select 40 premature infants admitted to the neonatology department of our hospital between January 2015 and December 2016. They were randomly divided into a BPD group and a control group. There were 20 premature infants in the BPD group and 20 premature infants in the control group. The BPD group included premature infants who continued to inhale oxygen for more than 28 days after birth and had a gestational age of less than 32 weeks. The inclusion criteria 
of the control group were as follows: (I) premature infants with gestational age less than 32 weeks; (II) an inhaled oxygen time of less than 3 days; and (III) an inhaled oxygen concentration of less than $30 \%$. Patients were excluded based on the following criteria: (I) premature infants with perinatal infection; (II) those with complex congenital heart disease; and (III) those with multiple malformations. A total of 15 cases were excluded.

\section{Specimen collection}

All blood samples were venous blood remaining after routine tests during hospitalization of the premature infants. The blood collection time of premature infants in the BPD group was 28 days after oxygen inhalation (the specific oxygen inhalation methods included air oxygen mixed nasal cannula oxygenation, continuous positive airway pressure (CPAP), or invasive ventilator assisted ventilation). The blood collection time of the premature infants in the control group was approximately 3 days after birth.

\section{Cell culture and screening}

A549 cells were obtained from American type culture collection (ATCC; America; we used the following cell lines: A549, catalog number: CCL-185), and were cultured in Dulbecco's Modified Eagle Medium (DMEM) (HyClone; GE Healthcare Life Sciences; China) containing $100 \mathrm{U} / \mathrm{mL}$ penicillin and $100 \mu \mathrm{g} / \mathrm{mL}$ streptomycin (Beijing Solarbio Science \& Technology Co., Ltd.; China) and 10\% (v/v) fetal bovine serum (FBS; Gibco; Thermo Fisher Scientific, Inc.; Australia). Cells in this complete culture medium were cultured to form a monolayer at $37{ }^{\circ} \mathrm{C}$ with $5 \%$ carbon dioxide $\left(\mathrm{CO}_{2}\right)$. The solutions were changed every 2-3 days based on the cells, growth and the change of the culture solution. When the cells grew to approximately $90 \%$ of the culture flask area, they were passaged or frozen. Well-grown cells from the 3 rd to the 10th generation were selected for this experiment. Before the cell intervention experiment, the cells were starved with DMEM medium (containing 10\% fetal bovine serum $+1 \%$ double antibody) for 12 to $24 \mathrm{~h}$.

\section{Cell transfection}

Before transfection, $5 \times 10^{4}$ cells were seeded on a 24 -well plate, and DMEM cell culture medium containing $10 \%$ FBS and antibiotics was added. The number of cells selected for initial inoculation should make the cell confluence reach $40-70 \%$ within 24 hours. Subsequently, 50, 100, and $200 \mathrm{nmol} / \mathrm{L}$ small interfering RNA (siRNA) were added to $50 \mu \mathrm{L}$ of DMEM serum-free medium and mixed gently. Dilute $1 \mu \mathrm{L}$ lipofectamin2000 reagent was mixed with $50 \mu \mathrm{L}$ serum-free DMEM [note: for deoxyribonucleic acid (DNA) transfection, add $2.4 \mu \mathrm{g}$ of RNAi-Mate reagent (GenePharma; China)], mixed gently, and left at room temperature for 5 minutes. Next, the diluted siRNA and lipofectamin reagent were mixed gently and left at room temperature for 20 minutes to form a siRNA/lipofectamin (or DNA/lipofectamin) complex. This complex was then added to the wells of the culture plate containing the cells and medium, and the cell culture plate was gently shaken back and forth.

Following transfection of three different siRNA MALAT1 plasmids into the A549 lung cancer cells, the changes in the expression of MALAT1 messenger RNA (mRNA) levels were detected and compared, and the one with the best inhibitory effect was selected for subsequent experiments (Table 1).

Following the above steps, we used the A549 cells for two groups of experiments, which were divided into four types of cells: (I) air group (non-interference air group), (II) hyperoxia group (non-interference hyperoxia group), (III) NC group (control plasmid transfection air group), and (IV) siRNA group (air group after interference). The hyperoxia group was exposed to a highly pure gas mixture of $3 \mathrm{~L} / \mathrm{min} 92 \%$ oxygen $\left(\mathrm{O}_{2}\right)$ and $5 \% \mathrm{CO}_{2}$, and was sealed after 10 minutes. Each group were placed in an incubator $\left(37{ }^{\circ} \mathrm{C}, 5 \% \mathrm{CO}_{2}\right)$. After incubation for 48 hours, we extracted the total RNA from the A549 cells.

\section{Western Blot detected the expression of related proteins}

In two groups of A549 cell experiments, Western blotting was used to detect Nrf2 and Keap1 proteins' expression trends under different experimental conditions. For the interference group experiment, the cells proceeded to the next step after exposure to air or hyperoxia following transfection of the MALAT1 siRNA plasmid into A549 cells. For the non-interference group experiment, the cells proceeded to the next step after air or hyperoxia.

Image Lab Software (Bio-Rad; America; Version 5) was used for analysis. $100 \mathrm{uL}$ of cell lysate was added to each well, and the cells were collected to extract the nuclear and cytoplasmic proteins. $150 \mathrm{uL}$ of $4 \times$ loading buffer were added to $50 \mathrm{uL}$ of the collected protein, mixed, and then 
Table 1 Primer of siRNA-1, siRNA-2 and siRNA-3

\begin{tabular}{lll}
\hline Gene name & Primer & Sequence (5'-3') \\
\hline siRNA-1 & Forward & GGAAGUAAUUCAAGAUCAAGA \\
& Reverse & UUGAUCUUGAAUUAUUCCGU \\
siRNA-2 & Forward & GCGACGAGUUGUGCUGCUAUC \\
& Reverse & UAGCAGCACAACUCGUCGCUG \\
SiRNA-2 & Forward & GAGUUGUGCUGCUAUCUUAGC \\
& Reverse & UAAGAUAGCAGCACAACUCGU \\
\hline
\end{tabular}

siRNA, small interfering RNA.

Table 2 Primer of MALAT1, AIF, $\beta$-actin $(\mathrm{H})$, Keap1, Nrf2 and GAPDH

\begin{tabular}{lll}
\hline Gene name & Primer & Sequence (5'-3') \\
\hline MALAT1 & Forward & AAAGCAAGGTCTCCCCACAAG \\
& Reverse & GGTCTGTGCTAGATCAAAAGGCA \\
AIF & Forward & CTGTTGTGAGTGGAAGATTGG \\
& Reverse & CAACTGTGGGCAAACTACTGT \\
$\beta$-actin $(H)$ & Forward & GTGGCCGAGGACTTTGATTG \\
& Reverse & CCTGTAACAACGCATCTCATATT \\
Keap1 & Forward & TGCGCTGCGAGTCCGAGGTCTTC \\
& Reverse & TCGAAGATCTTGACCAGGTAGT \\
Nrf2 & Forward & ATGGATTTGATTGACATACTTT \\
& Reverse & ACTGAGCCTGATTAGTAGCAAT \\
GAPDH & Forward & CATCACTGCCACCCAGAAGACTG \\
& Reverse & ATGCCAGTGAGCTTCCCGTTCAG \\
\hline
\end{tabular}

MALAT1, metastasis-associated lung adenocarcinoma transcript 1; AIF, apoptosis-induced factor; Keap1, Kelch-like ECHassociated protein 1; Nrf2, nuclear factor erythroid-2-related factor 2; GAPDH, glyceraldehyde-3-phosphate dehydrogenase.

incubated at $100{ }^{\circ} \mathrm{C}$ for 5 minutes and cooled on ice. The separation gel and concentrated gel were configured, and $40 \mathrm{uL}$ was loaded per well, which was run for 90 minutes. After completion of the transfer, the power was turned off, and the polyvinylidene fluoride (PVDF) membrane was removed and placed in a container (dish). An appropriate amount of blocking buffer [phosphate-buffered saline (PBS) containing 5\% (w/v) skimmed milk powder] was added, and was gently shaken at room temperature for 1 to 2 hours. This was then replaced with a new culture dish, and $10 \mathrm{ml}$ of the above blocking buffer and the primary antibody [MALAT1 (Biorbyt Cat\#orb500388; China), Keap1 (Abcam Cat\#ab227828; England), and Nrf2 (Abcam Cat\#ab62352; England)] were added at a ratio of 1:1000. The culture was incubated at $4{ }^{\circ} \mathrm{C}$ overnight, and was subsequently washed three times with PBS buffer for 10 min each time. The PVDF membrane was then transferred to another petri dish, and secondary antibody buffer (containing 5\% skimmed milk powder) and the secondary antibody [horseradish peroxidase (HRP)labeled goat anti-rabbit immunoglobulin $\mathrm{G}(\mathrm{IgG})$ ] were added at a ratio of 1:5000 and incubated for 1 hour at room temperature with shaking. The PVDF membrane was subsequently transferred to another culture dish, and an appropriate amount of secondary antibody buffer [150 mM sodium chloride $(\mathrm{NaCl}), 50 \mathrm{mM}$ Tris-Cl $\mathrm{pH}$ 7.5] was added and washed three times at room temperature for 10 min each time. Electrochemiluminescence (ECL) substrate developer (Thermo) and the substrate developer were added for imaging.

\section{Total RNA isolation and real-time polymerase chain reaction (PCR) verification}

According to the manufacturer's instructions, total RNA was extracted from the 40 premature infants' venous blood using TRIzol reagent (Invitrogen, Carlsbad, USA) according to the BPD and control groups. NanoDrop ND-2000 Spectrophotometer (NanoDrop, Wilmington, DE) was used to quantify the RNA. We have detailed descriptions of the reverse transcription (RT) reaction and real-time PCR experiments (19). The $2-\Delta \Delta \mathrm{Ct}$ method was used to calculate the relative expression of MALAT1 and AIF compared to $\beta$-actin (H) (Table 2).

For the A549 cell experiment, the medium was aspirated from the 6-well plate, added to the 6 -well plate with $1 \times \mathrm{PBS}$, washed gently, and then placed on ice. Next, $800 \mu \mathrm{L}$ of TRIzol was added and all adherent cells were repeatedly pipetted. It was then beat down and transferred to a $1.5 \mathrm{~mL}$ Eppendorf (EP) tube. $200 \mu \mathrm{L}$ of chloroform was added and shaken vigorously. This was then centrifuged at $12,000 \mathrm{rpm}$ at $4{ }^{\circ} \mathrm{C}$ for 5 minutes. The supernatant was subsequently transferred to a $1.5 \mathrm{~mL}$ small tube, and the same volume (as the supernatant) of isopropanol was added. It was then left at $-20^{\circ} \mathrm{C}$ for 1 hour or longer (until fully precipitated). To avoid DNA contamination, we did not suck the middle layer material. This was subsequently centrifuged at $4{ }^{\circ} \mathrm{C}$ at $12,000 \mathrm{rpm}$ for $5 \mathrm{~min}$. We then carefully removed the supernatant to prevent the loss of precipitation, washed 
Table 3 Compare gestational age and birth weight of preterm infants in both groups

\begin{tabular}{lccc}
\hline Group & $\mathrm{n}$ & $\begin{array}{c}\text { Gestational age } \\
\text { (week) }\end{array}$ & birth weight $(\mathrm{g})$ \\
\hline Control group & 20 & $29.1 \pm 2.47$ & $1,620 \pm 289$ \\
BPD group & 20 & $28.8 \pm 4.26$ & $1,570 \pm 335$ \\
$\mathrm{t}$ & & 0.169 & 1.247 \\
$\mathrm{P}$ value & & 0.418 & 0.326 \\
\hline
\end{tabular}

BPD, bronchopulmonary dysplasia.

Table 4 Real-time PCR was used to detect and compare the expression of MALAT1 and AIF in preterm infants

\begin{tabular}{lccc}
\hline Group & $\mathrm{n}$ & MALAT1 & AlF \\
\hline Control group & 20 & $0.2734 \pm 0.0673$ & $0.0068 \pm 0.0020$ \\
BPD group & 20 & $0.3755 \pm 0.0819$ & $0.0045 \pm 0.0019$ \\
$\mathrm{t}$ & & 4.635 & 3.147 \\
$\mathrm{P}$ value & & 0.015 & 0.022 \\
\hline
\end{tabular}

MALAT1, metastasis-associated lung adenocarcinoma transcript 1; AIF, apoptosis-induced factor; BPD, bronchopulmonary dysplasia.

it twice with $70 \%$ ethanol ( $700 \mu \mathrm{L}$ each time). This was centrifuged at $4{ }^{\circ} \mathrm{C}$ at $12,000 \mathrm{rpm}$ for $5 \mathrm{~min}$. We carefully sucked up the supernatant as much as possible, and inverted the alcohol on a clean bench to dry it. Finally, $30 \mu \mathrm{L}$ of diethypyrocarbonate (DEPC) treated water was added, and it was then stored at $-70^{\circ} \mathrm{C}$.

A549 cells of the siRNA group were transfected with the MALAT1 siRNA plasmid. The expression levels of MALAT1, Keap1, and Nrf2 mRNA were detected by realtime PCR. The relative expression of MALAT1, Keap1, and Nrf2 compared to glyceraldehyde-3-phosphate dehydrogenase (GAPDH) was calculated using the $2-\Delta \Delta \mathrm{Ct}$ method (Table 2).

\section{Measurement of cell apoptosis by flow cytometry}

After intervention of hyperoxia and siRNA targeting MALAT1, the apoptosis of A549 cells was detected using the Annexin V-FITC apoptosis kit (BD; America). Specific procedures for the reagents used to detect apoptosis and the experiments have been discussed (20).

\section{Statistical analysis}

The experimental data were statistically analyzed using SPSS 23.0 statistical software (Armonk, NY: IBM Corp), and the results represented the mean \pm standard deviation (SD) of the independent experiments. The data between the two groups were compared using two independent samples $t$-tests, and $\mathrm{P}<0.05$ was considered statistically significant.

\section{Results}

\section{Basic information}

Among the 40 premature infants, 26 were male and 14 were female, with an average gestational age of $29.0 \pm 3.64$ weeks. There were 24 cases of birth weight $\leq 1,500 \mathrm{~g}$, and 16 cases between 1,500 and 2,500 $\mathrm{g}$, and all of them are premature infants. There were no perinatal infections with or without neonatal respiratory distress syndrome (NRDS), and their admission time was less than 12 hours after birth. There was no significant difference in gestational age $(\mathrm{t}=0.169, \mathrm{P}=0.418)$ and birth weight between the two groups ( $\mathrm{t}=1.247, \mathrm{P}=0.326$ ) (Table 3).

\section{Detection of MALAT1 and AIF expression by real-time PCR}

Compared with that of the control group, the expression of MALAT1 in the BPD group increased significantly $(\mathrm{P}<0.05)$, while the expression of AIF in the BPD group markedly decreased $(\mathrm{P}<0.05)$ (Table 4 and Figure 1).

\section{MALAT1 suppression by siRNA}

SiRNA-2 had the highest inhibitory efficiency. The results showed that the inhibition efficiency was the highest when the siRNA plasmid was transfected into the cells for 48 hours, and thus, 48 hours was selected as the time point for followup of the transfection experiment (Table 5 and Figure 2).

\section{Detection of relative expression of MALAT1, Keap1 and Nvf2 exposed to byperoxia}

Compared with that of the air group, the expression of MALAT1 in the hyperoxia group decreased considerably $(\mathrm{P}<0.05)$, while the expression of Keap1 and Nrf2 in the non-interference hyperoxia group increased substantially $(\mathrm{P}<0.05)$ (Table 6, Figures 3,4). 

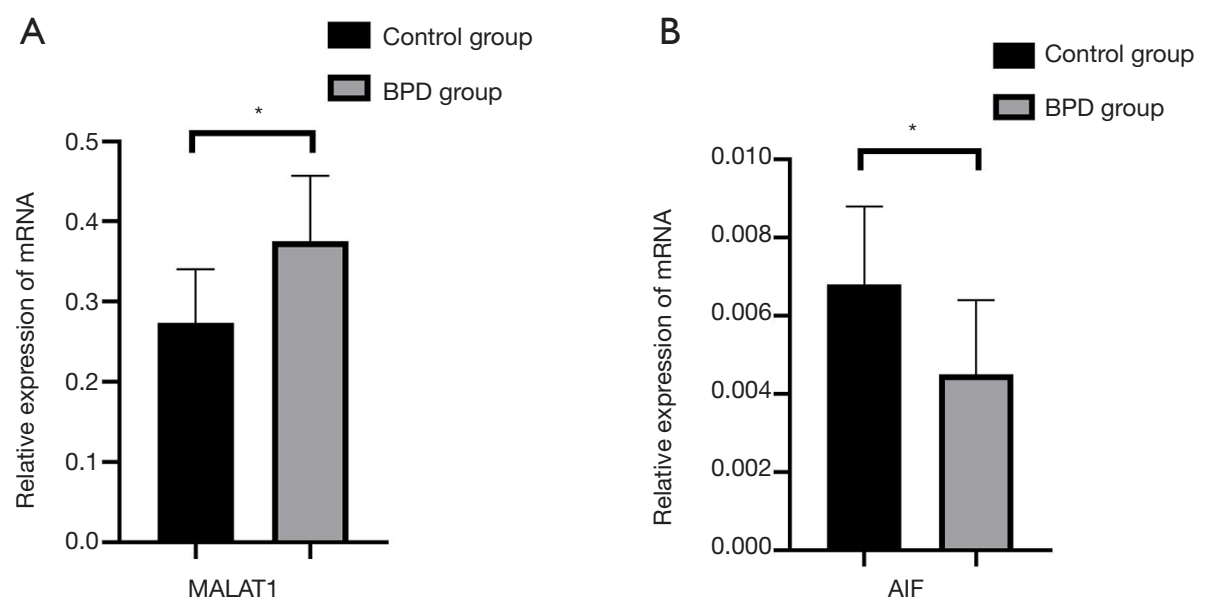

Figure 1 Comparison of the expression of MALAT1 and AIF in preterm infants. Compared with that of the control group, the expression of MALAT1 in the BPD group increased (A), while the expression of AIF decreased (B). Data represents the mean \pm standard deviation (SD). ${ }^{*} \mathrm{P}<0.05$.

Table 5 Detection and screening of the most efficient target for siRNA silencing MALAT1 by RT-PCR

\begin{tabular}{llll}
\hline$N C(n=3)$ & siRNA-1 $(n=3)$ & siRNA-2 $(n=3)$ & siRNA-3 $(n=3)$ \\
\hline $1.0362 \pm 0.0750$ & $0.3614 \pm 0.0445$ & $0.2874 \pm 0.0706$ & $0.6343 \pm 0.1836$ \\
\hline
\end{tabular}

siRNA, small interfering RNA; MALAT1, metastasis-associated lung adenocarcinoma transcript 1; RT-PCR, real-time polymerase chain reaction; NC, control plasmid transfection group.

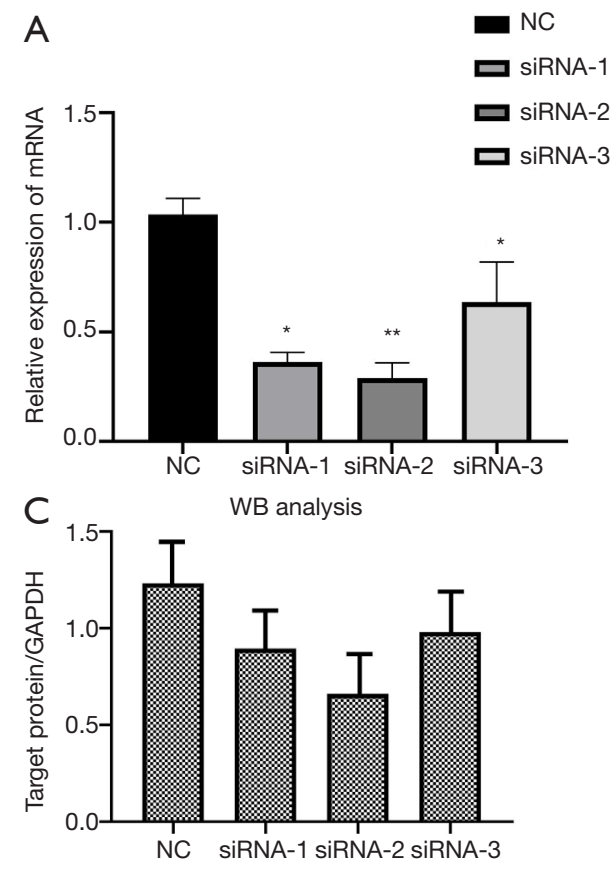

B
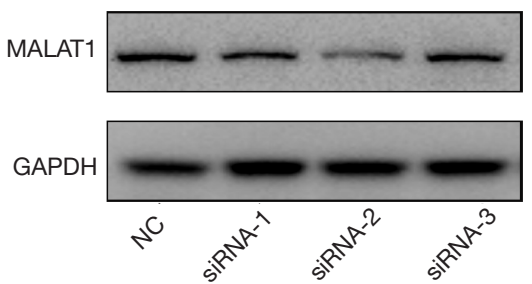

Figure 2 Comparison of the effects of siRNA in inhibiting MALAT1. MALAT1 was suppressed by siRNA. siRNA was designed by professional company (GenePharma; China). MALAT1 not suppressed by siRNA in the control group. siRNA1, siRNA2, and siRNA3 represent three different siRNAs transfected into A549 cells $(\mathrm{A}, \mathrm{B}, \mathrm{C})$. Data represents the mean $\pm \mathrm{SD}$. ${ }^{*} \mathrm{P}<0.01,{ }^{*} \mathrm{P}<0.05$. 
Table 6 Effect of hyperoxia on the expression of MALAT1, Keap1 and Nrf2 in A549 cells

\begin{tabular}{lcccc}
\hline Group & $\mathrm{n}$ & MALAT1 & Keap1 & Nrf2 \\
\hline Air & 3 & $0.9633 \pm 0.0543$ & $1.0247 \pm 0.0630$ & $1.0028 \pm 0.0929$ \\
Hyperoxia & 3 & $0.3993 \pm 0.0557$ & $3.2991 \pm 0.4834$ & $4.5525 \pm 0.4978$ \\
$\mathrm{t}$ & & 12.559 & -8.081 & -12.140 \\
$\mathrm{P}$ value & & $<0.001$ & 0.001 & $<0.001$ \\
\hline
\end{tabular}

MALAT1, metastasis-associated lung adenocarcinoma transcript 1; Keap1, Kelch-like ECH-associated protein 1; Nrf2, nuclear factor erythroid-2-related factor 2 .
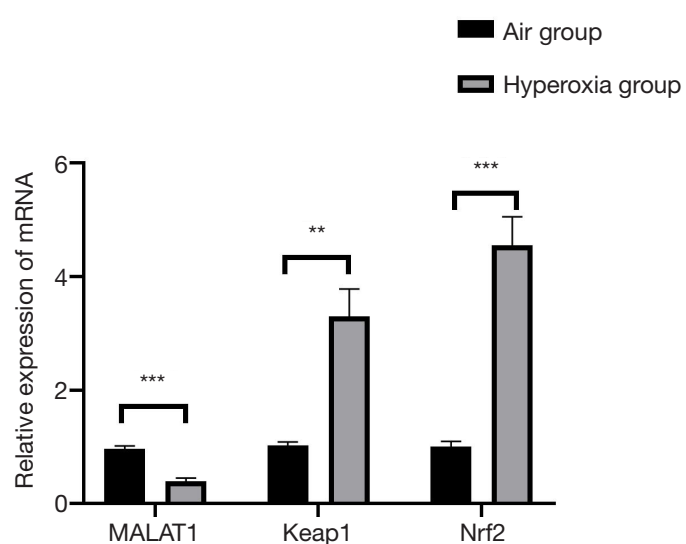

Figure 3 Comparison of the expression of MALAT1, Keap1, and Nrf2 following exposure to hyperoxia. Compared with that of the air group, the expression of MALAT1 in the hyperoxia group decreased significantly, while the expression of Keap1 and Nrf2 increased considerably (Figure 3). Data represents the mean \pm SD. ${ }^{* *} \mathrm{P}<0.01,{ }^{* * *} \mathrm{P}<0.001$.

\section{Detection of relative expression of MALAT1, Keap1, and Nrf2 after MALAT1 inbibition by siRNA}

Compared with that of the NC group, the expression of MALAT1 in the siRNA group decreased significantly $(\mathrm{P}<0.05)$, while the expression of Keap1 and Nrf2 increased markedly $(\mathrm{P}<0.05)$ (Table 7, Figures 4,5).

\section{Inbibition of MALAT1 gene exposed to hyperoxia promotes apoptosis}

After silencing MALAT1 with siRNA, the percentage of early and late apoptosis in A549 cells in the hyperoxia group was decreased after $0,24,48$, and 72 hours interference, and was as follows: $29.990 \pm 0.834 v s .7 .985 \pm 0.530(0 \mathrm{~h})$, $28.360 \pm 0.339$ vs. $7.215 \pm 0.064$ ( $24 \mathrm{~h}), 27.725 \pm 0.813$ vs.
$6.555 \pm 0.346(48 \mathrm{~h})$, and $11.250 \pm 1.768$ vs. $4.415 \pm 0.290(72 \mathrm{~h})$ (Figure 6).

\section{Discussion}

To date, not much information is available in the literature regarding the function of MALAT1 in premature infants with BPD and its role in developing BPD. Oxygen inhalation is an important treatment for respiratory failure in premature infants, however long-term hyperoxia exposure can cause acute lung injury, which ultimately contributes to the occurrence of BPD (21). It is currently believed that the imbalance of the oxidation/antioxidation mechanism in the body is one of BPD's pathogenesis mechanisms (22). There is a lack of specific drugs and measures for the prevention and treatment of BPD. Therefore, investigating BPD's pathogenesis, identifying molecular markers for the early warning of $\mathrm{BPD}$, and preventing the occurrence of BPD are some of the challenges faced by neonatologists.

In this study, the expression of MALAT1 in the BPD group increased significantly compared with that of the control group. This finding is consistent with that of Cai et al. who reported that MALAT1 is significantly upregulated in the lung tissue of BPD mice (23). In the A549 cell model, we found that the expression of MALAT1 in the hyperoxia group was opposite to that in the BPD group of premature infants, likely because the cells showed a transient reduction in MALAT1 without the control of the intact organism. This may gradually increase later under the control of the body. Studies have confirmed that MALAT1 can inhibit apoptosis and promote cell proliferation, suggesting that MALAT1 may play an important protective role during the development of BPD.

Over-expression of AIF results in peripheral chromatin condensation, mitochondrial transmembrane potential dissipation, and DNA degradation. Qu et al. found that 


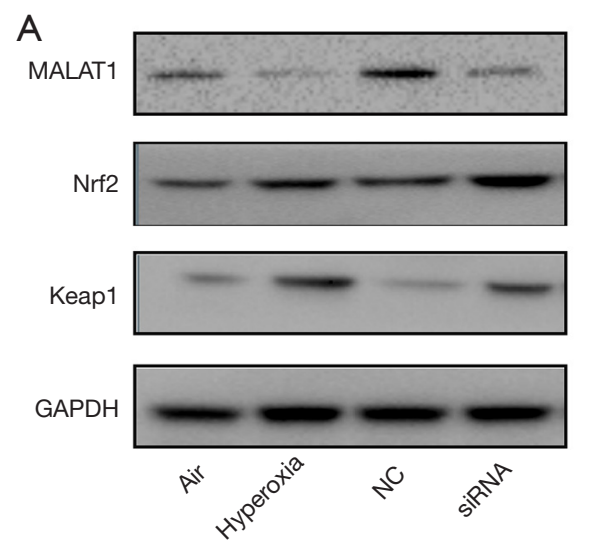

B WB analysis
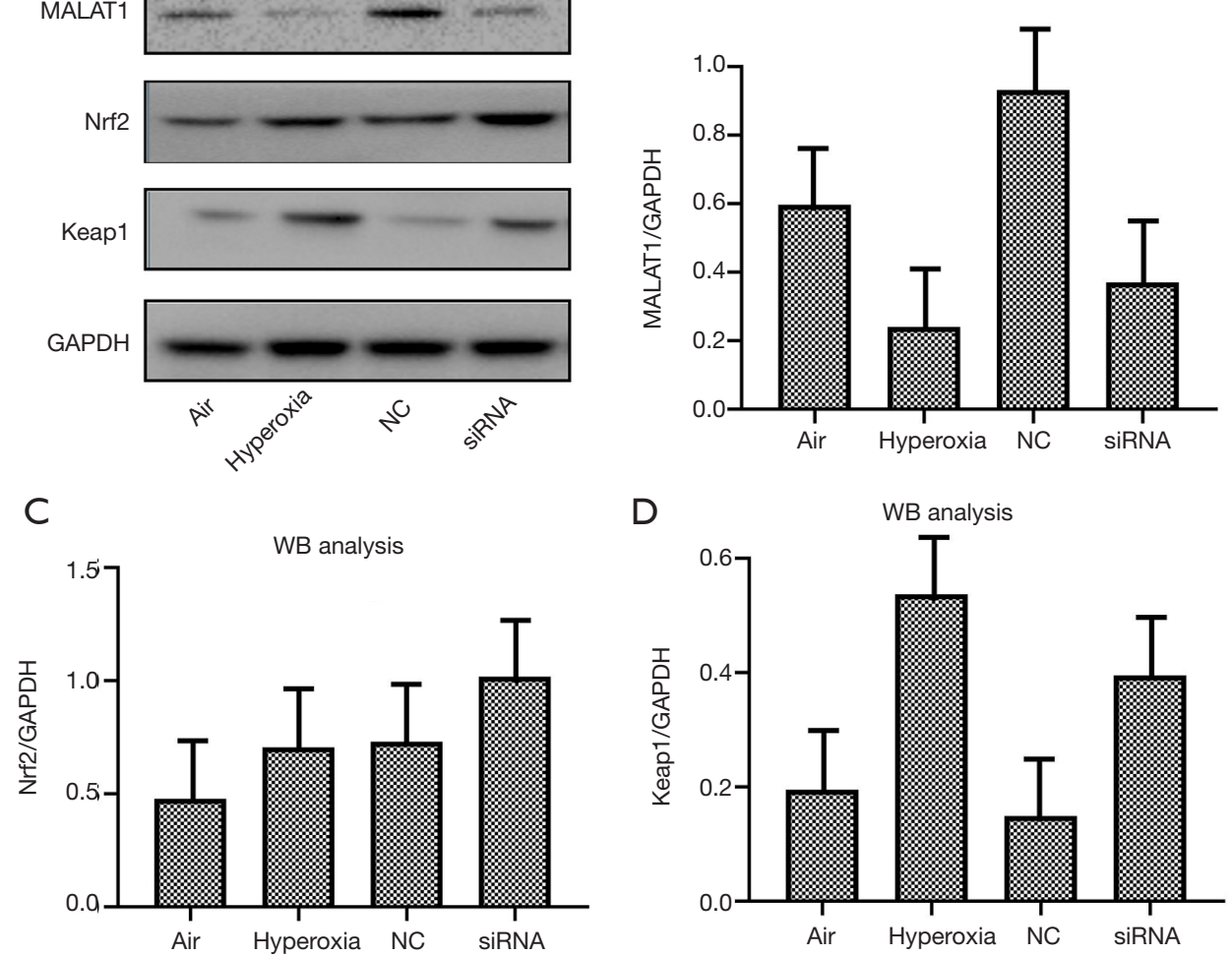

Figure 4 Comparison of the expression of MALAT1, Keap1, and Nrf2. A549 cells were exposed to hyperoxia and subsequently subjected to protein isolation. MALAT1 was suppressed by siRNA and subsequently subjected to protein isolation. All target genes and GAPDH (as a reference gene) were measured using Western blot analysis.

Table 7 Effect of siRNA silencing MALAT1 on the expression of MALAT1, Keap1 and Nrf2 in A549 cells

\begin{tabular}{lcccc}
\hline Group & $\mathrm{n}$ & MALAT1 & Keap1 & Nrf2 \\
\hline $\mathrm{NC}$ & 3 & $0.9885 \pm 0.1246$ & $1.0277 \pm 0.0603$ & $1.0490 \pm 0.1363$ \\
siRNA & 3 & $0.3011 \pm 0.0762$ & $1.2869 \pm 0.1171$ & $1.5787 \pm 0.2116$ \\
$\mathrm{t}$ & & 8.151 & -3.410 & -3.645 \\
$\mathrm{P}$ value & & 0.001 & 0.027 & 0.022 \\
\hline
\end{tabular}

siRNA, small interfering RNA; MALAT1, metastasis-associated lung adenocarcinoma transcript 1; Keap1, Kelch-like ECH-associated protein 1; Nrf2, nuclear factor erythroid-2-related factor 2; NC, control plasmid transfection air group.

inhibition of AIF expression by siRNA can partially reduce apoptosis in the A549 human alveolar epithelial cell line (24). Therefore, we hypothesized that the low expression of AIF in the BPD group may impede cell growth by inhibiting mitochondrial activity (mitochondrial biosynthesis and oxidative phosphorylation) limiting part of the cell's energy source. This suggests that AIF may be involved in developing BPD by regulating mitochondrial homeostasis or cellular metabolism.
Ma et al. noted that under high oxygen conditions, the expression of Keap1 decreased and the expression of Nrf2 increased (25). However, we found that in a hyperoxic environment, the expression of Keap1 and Nrf2 both increased significantly, which could exert anti-inflammatory and anti-oxidation effects (26).

According to Zeng et al., the inhibition of MALAT1 expression by siRNA resulted in up-regulation of Keap1 expression in human umbilical vein endothelial cells and 


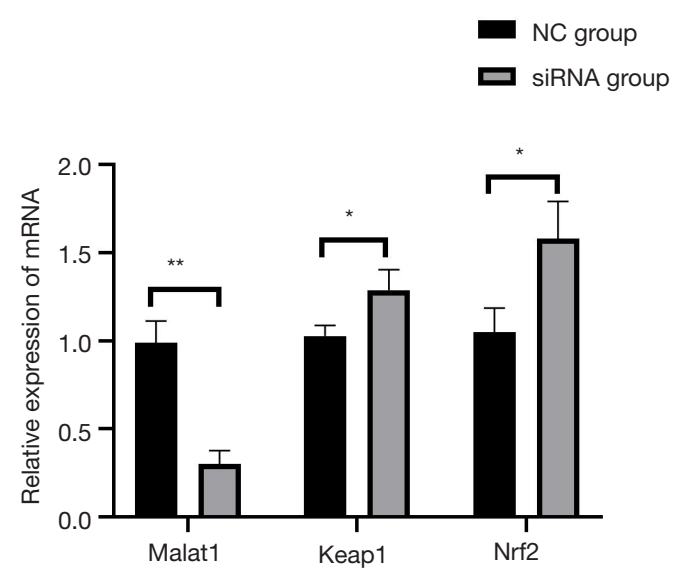

Figure 5 Comparison of the expression of MALAT1, Keap1, and Nrf2 after silencing of MALAT1. Compared with that of the NC group, the expression of MALAT1 in the siRNA group decreased significantly, while the expression of Keap1 and Nrf2 markedly increased. Data represents the mean $\pm \mathrm{SD} .{ }^{*} \mathrm{P}<0.05,{ }^{* *} \mathrm{P}<0.01$. decreased expression of the Nrf2 protein (27). Regarding the expression of Keap1 after silencing the expression of MALAT1 (siRNA group), our experimental results were contrary to those of Zeng et al., probably because the experimental models were inconsistent. However, our results for the expression trend of Nrf2 were consistent with Zeng et al.

We hypothesized that MALAT1 and the Keap1/Nrf2 signaling pathway play a protective role in BPD through interaction, with the former mainly exerting anti-apoptotic effect, and the latter primarily playing an antioxidant role. When MALAT1 is inhibited (siRNA group), the Keap1/ Nrf2 signaling pathway can play a compensatory role, and their common goal is to protect BPD premature infants from antagonizing apoptosis and/or anti-oxidation.

After silencing MALAT1 with siRNA exposed to hyperoxia, the percentage of apoptosis in A549 cells was gradually and significantly increased. By studying A549 cells
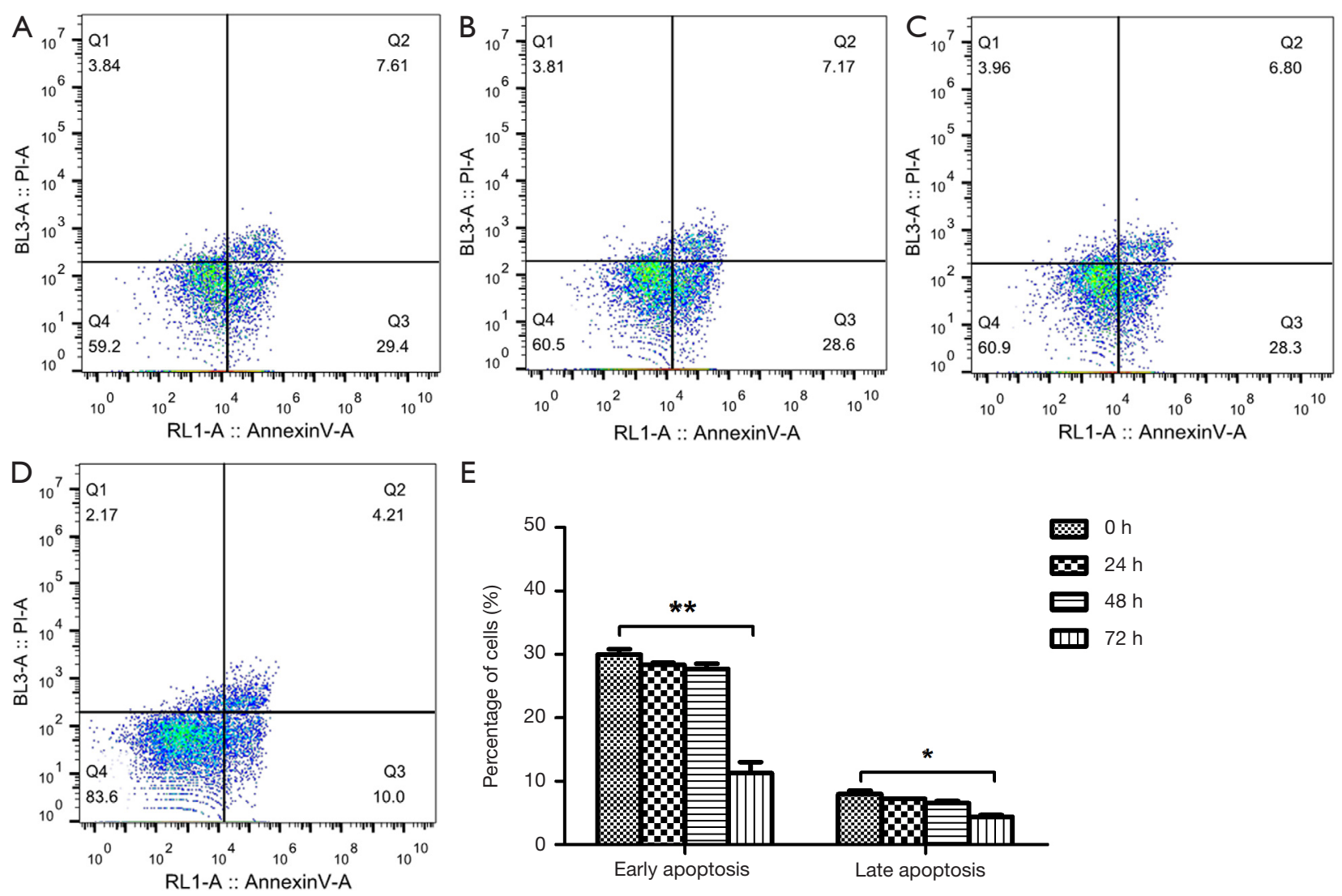

Figure 6 Apoptosis of A549 cells exposed to hyperoxia for 0, 24, 48 and $72 \mathrm{~h}$ after silencing of MALAT1. As time progressed, the apoptosis rate of the cells gradually decreased, and these were $29.990 \pm 0.834$ vs. $7.985 \pm 0.530(0 \mathrm{~h}), 28.360 \pm 0.339 v s .7 .215 \pm 0.064(24 \mathrm{~h}), 27.725 \pm 0.813$ vs. $6.555 \pm 0.346$ (48 h), and $11.250 \pm 1.768$ vs. $4.415 \pm 0.290$ (72 h) (A,B,C,D,E). ${ }^{*} \mathrm{P}<0.05 ;{ }^{* *} \mathrm{P}<0.01$. 
transfected with short hairpin metastasis-associated lung adenocarcinoma transcript 1 (shMALAT1) and shRNA, Cai et al. found that the amount of apoptosis was considerably increased, which indicates that down-regulation of MALAT1 promotes apoptosis (23). We can speculate that although the Keap1/Nrf2 signaling pathway can compensate for the anti-oxidation effect after MALAT1 is knocked out (siRNA group), it still cannot prevent cell apoptosis, which demonstrates that MALAT1 plays a dominant protective role in BPD.

Our research has some limitations that should be noted. Firstly, the detection of serum MALAT1 and AIF levels may have some reference value for the early diagnosis of BPD, but the sample size in this study is small, and thus, further large sample size multi-center studies are needed to verify the significance of MALAT1 and AIF in indicating BPD. Secondly, we originally planned to use WI-38 cells for experimental verification, however these cells are difficult to obtain and culture. Although rodent type II cells are more readily available, they are still different from human type II cells.

\section{Conclusions}

Apoptosis and oxidative stress are two key aspects of BPD development. If the apoptosis and oxidative stress during the occurrence of BPD can be reduced, BPD can be effectively controlled. Through our experiments, we have shown that MALAT1 plays a protective role in BPD via the inhibition of apoptosis and antioxidant effects, which provides new ideas for the prevention and treatment of BPD.

\section{Acknowledgments}

Funding: This study was funded by the National Natural Science Foundation of China (81571467).

\section{Footnote}

Reporting Checklist: The authors have completed the MDAR checklist. Available at http://dx.doi.org/10.21037/tp-20-200

Data Sharing Statement: Available at http://dx.doi. org/10.21037/tp-20-200

Conflicts of Interest: All authors have completed the International Committee of Medical Journal Editors (ICMJE) uniform disclosure form (available at http://dx.doi. org/10.21037/tp-20-200). The authors have no conflicts of interest to declare.

Ethical Statement: The authors are accountable for all aspects of the work in ensuring that questions related to the accuracy or integrity of any part of the work are appropriately investigated and resolved. This study was conducted following the Declaration of Helsinki (as revised in 2013), and was approved by the committee board of the Shanghai Children's Hospital (No.: 2015RY009-F01). Informed consent was obtained from the parents of all included patients.

Open Access Statement: This is an Open Access article distributed in accordance with the Creative Commons Attribution-NonCommercial-NoDerivs 4.0 International License (CC BY-NC-ND 4.0), which permits the noncommercial replication and distribution of the article with the strict proviso that no changes or edits are made and the original work is properly cited (including links to both the formal publication through the relevant DOI and the license). See: https://creativecommons.org/licenses/by-nc-nd/4.0/.

\section{References}

1. Collins JJP, Tibboel D, de Kleer IM, et al. The future of bronchopulmonary dysplasia: emerging pathophysiological concepts and potential new avenues of treatment. Front Med (Lausanne) 2017;4:61.

2. Horbar JD, Carpenter JH, Badger GJ, et al. Mortality and neonatal morbidity among infants 501 to 1500 grams from 2000 to 2009. Pediatrics 2012;129:1019-26.

3. Prasanth KV, Spector DL. Eukaryotic regulatory RNAs: an answer to the 'genome complexity' conundrum. Genes Dev 2007;21:11-42.

4. Ji P, Diederichs S, Wang W, et al. MALAT-1, a novel noncoding RNA, and thymosin beta4 predict metastasis and survival in earlystage non-small cell lung cancer. Oncogene 2003;22:8031-41.

5. Yoshimoto R, Mayeda A, Yoshida M, et al. MALAT1 long non-coding RNA in cancer. Biochim Biophys Acta 2016;1859:192-9.

6. Michalik KM, You X, Manavski Y, et al. Long noncoding RNA MALAT1 regulates endothelial cell function andvessel growth. Circ Res 2014;114:1389-97.

7. Li X, Song Y, Liu F, et al. Long Non-Coding RNA MALAT1 Promotes Proliferation, Angiogenesis, and Immunosuppressive Properties of Mesenchymal Stem 
Cells by Inducing VEGF and IDO.J Cell Biochem 2017;118:2780-91.

8. Liu H, Wang H, Wu B, et al. Down-regulation of long non-coding RNA MALAT1 by RNA interference inhibits proliferation and induces apoptosis in multiple myeloma. Clin Exp Pharmacol Physiol 2017;44:1032-41.

9. Wu L, Wang X, Guo Y. Long Non-Coding RNA MALAT1 is upregulated and involved in cell proliferation migration and apoptosis in ovarian cancer. Exp Ther Med 2017;13:3055-60.

10. Schmidt LH, Spieker T, Koschmieder S, et al. The long non coding MALAT-1 RNA indicates a poor prognosis in non-small cell lung cancer and induces migration and tumor growth. J Thorac Oncol 2011;6:1984-92.

11. Susin SA, Lorenzo HK, Zamzami N, et al. Molecular characterization of mitochondrial apoptosis-inducing factor. Nature 1999;397:441-6.

12. Agarwal E, Chaudhuri A, Leiphrakpam PD, et al. Akt inhibitor MK-2206 promotes anti-tumor activity and cell death by modulation of AIF and Ezrin in colorectal cancer. BMC Cancer 2014;14:145.

13. Urbano A, Lakshmanan U, Choo PH, et al. AIF suppresses chemical stress-induced apoptosis and maintains the transformed state of tumor cells. EMBO J 2005;24:2815-26.

14. Bano D, Prehn JHM. Apoptosis-Inducing Factor (AIF) in Physiology and Disease: The Tale of a Repented Natural BornKiller. EBioMedicine 2018;30:29-37.

15. Taguchi K, Motohashi H, Yamamoto M. Molecular mechanisms of the Keapl-Nrf2 pathway in stress response and cancer evolution. Genes Cells 2011;16:123-40.

16. Sun Z, Zhang S, Chan JY, et al. Keap1 Controls postinduction repression of the Nrf2- mediated antioxidant response by escorting nuclear export of Nrf2. Mol Cell Biol 2007;27:6334-49.

17. Jiang $\mathrm{T}$, Huang $\mathrm{Z}$, Lin $\mathrm{Y}$, et al. The protective role of $\mathrm{Nrf} 2$ in streptozotocin -induced diabetic nephropathy. Diabetes 2010;59:850-60.

Cite this article as: Zhang $M$, Zhang $\mathrm{X}$, Chu X, Cheng L, Cai C. Long non-coding RNA MALAT1 plays a protective role in bronchopulmonary dysplasia via the inhibition of apoptosis and interaction with the Keap1/Nrf2 signal pathway. Transl Pediatr 2021;10(2):265-275. doi: 10.21037/tp-20-200
18. Jeong WS, Jun M, Kong AN. Nrf2: A potential molecular target for cancer chemoprevention by natural compounds. Antioxid Redox Signal 2006;8:99-106.

19. Cai C, Qiu G, Gong X, et al. Effects of erythromycin on $\gamma$-glutamyl cysteine synthetase and interleukin-1 $\beta$ in hyperoxia-exposed lung tissue of premature newborn mices. J Pediatr (Rio J) 2014;90:493-9.

20. Zhang $X$, Chu X, Gong $X$, et al. The expression of miR125b in Nrf2-silenced A549 cells exposed to hyperoxia and its relationship with apoptosis. J Cell Mol Med 2020;24:965-72.

21. Buczynski BW, Maduekwe ET, O'Reilly MA. The role of hyperoxia in the pathogenesis of experimental BPD. Semin Perinatol 2013;37:69-78.

22. Silva DM, Nardiello C, Pozarska A, et al. Recent advances in the mechanisms of lung alveolarization and the pathogenesis of bronchopulmonary dysplasia. Am J Physiol Lung Cell Mol Physiol 2015;309:L1239-72.

23. Cai C, Qiu J, Qiu G, et al. Long non-coding RNA MALAT1 protects preterm infants with bronchopulmonary dysplasia by inhibiting cell apoptosis. BMC Pulm Med 2017;17:199.

24. Qu X, Ding X, Duan M, et al. Influenza virus infection induces translocation of apoptosis-inducing factor (AIF) in A549 cells: roll of AIF in apoptosis and viral propagation. Arch Virol 2017;162:669-75.

25. Ma D, Gao W, Liu J, et al. Mechanism of oxidative stress and Keap-1/Nrf2 signaling pathway in bronchopulmonary dysplasia. Medicine (Baltimore) 202026;99:e20433.

26. Tu W, Wang H, Li S, et al. The Anti-Inflammatory and Anti-Oxidant Mechanisms of the Keap1/Nrf2/ ARE Signaling Pathway in Chronic Diseases. Aging Dis 2019;10:637-51.

27. Zeng $\mathrm{R}$, Zhang $\mathrm{R}$, Song $\mathrm{X}$, et al. The long non-coding RNA MALAT1 activates Nrf2 signaling to protect human umbilical vein endothelial cells from hydrogen peroxide. Biochem Biophys Res Commun 2018;495:2532-8. 